\title{
An Empirical Study of DEA in Developmental Teaching Evaluation
}

\author{
Chen Qiao ${ }^{1}$, Wu Xiujun ${ }^{2 *}$ \\ ${ }^{1,2}$ School of Mathematics and Computer Science, Jianghan University, Wuhan, Hubei, China \\ *Corresponding author-Emailwxj1818@sina.com
}

\begin{abstract}
Based on the conception of developmental evaluation, the teaching process is considered as a input-ouput system. For the purpose of teaching-learning co-promoting mode, the Data Envelopment Analysis method could effectively be used to evaluate the institutional teaching capacity, as well as carry out the empirical analysis. It is proved to be effective for the DEA model for promoting the development of diagnostic evaluation of teaching and learning.
\end{abstract}

Keywords: DEA model; teaching efficiency; evaluation model

\section{数据包络分析在发展性教学评价中的实证研究}

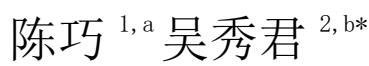

中国江汉大学, 数学与计算机科学学院, 武汉, 湖北

a1990642573@qq.com

${ }^{b}$ wxj1818@sina.com *通讯作者

\section{摘要}

基于发展性评价理念，把教师的教学过程看作是一个投入产出系统，以教学相长为目的，用数据包络 分析方法对学校教学水平进行有效评价，并进行了实证分析，该方法为教与学的良性发展诊断性评估 进行了积极的探索。

关键词: DEA 模型; 教学效率; 评价模型

\section{1. 问题的提出}

教学评价是指在教学过程中依据教学目标, 有计划、 有目的地观察、测定教师和学生学习的种种变化, 根据 这些变化对照教学目标、教学计划、教学效果、学生的 学习质量及个性发展水平, 运用科学的方法做出价值判 断, 进而调整、优化教学进程, 促进学生达成教学目标 的教学实践活动。综合各方观点, 可以把教育评价的概 念归结为: 依据一定的标准, 在系统、全面地收集、整 理和运用教育信息的基础上, 对教育活动的过程和结果 进行价值判断, 以做出相应改善和调整来促进教育活动 的过程 ${ }^{[1]}$ 。

目前学校对教师教学水平的评价, 更多的是用评 估、评审、考核等概念代替评价，其表现形式主要为： 职称评审制度、教师考核制度、阶段性评估制度、教学 督导等 ${ }^{[1]}$ 。从评价目的看, 按照评价的目的有静态评价 和动态评价两种方法, 静态的评价便于横向的比较, 区分出同一类别素质的差异, 以奖优罚劣。动态的评价 从发展的角度看待人的素质发展, 以帮助人才发展。学 校目前绝大多数采取的评价方法属于静态评价, 是面向 过去、注重结果的一种终结性评价, 这种评价方式的客
观性和科学性都面临着挑战, 不符合人才成长规律, 无法给教师的教学创新和学生发展提供自由的空间。

发展性教学评价是西方发达国家的先进的教学评 价方法，既注重对教师教学绩效的鉴定，又强调对教 师教学过程的评价。发展性教学评价以促进教师发展为 目的, 着眼未来的形成性评价。其通过评价确定教师教 学过程中的问题所在, 并对这些问题提出改进意见, 把评价的结果和意见及时反馈给教师，从而提高教师 的能力和水平。

新一轮基础教育课程改革明确提出, 要建立促进 学生、教师和课程不断发展的评价体系, 即建立发展性 课程评价体系. 这种评价体系的建立无疑具有重要的 理论与现实价值. 因为, 教育价值必然要回归到个体多 样化的发展，这种发展的前提是个体潜能的充分开发， 这种发展的核心是个体创造力的充分养成, 这种发展的 现实形态是个体自我的充分实现 ${ }^{[2][3]}$. 因此如何建立发 展性的学校教学评价体系成为一个现实议题。

随着我国经济快速发展的通知, 我国对于人才培养 也提出了一系列改革, 其中最显著的便是新课改的提出, 新课改中强调的教学效率这一用词更加吸引人眼球, 虽 然新课改在很大程度上提高了一部分质量, 但是教学效 率不高的问题依旧很突出, 这也使得我们必须建立一套 
教学效率评价体系。只有建立科学, 合理, 有效的评价 体系, 才能正确评价教学效率。评价结果不仅有助于教 师学习体会教学方法, 而且可以更加有效的利用课堂教 学时间, 同时减少学生的工作量并提高质量, 此外, 有 效执行课堂教学任务还可以使学生独立探索有效的学 习方法 ${ }^{[3]}$ 。

学校教学评价研究是新课改中的一项重要工作。在 国内外, 关于这一问题的大多数研究都是从不同的角度, 以各种研究方法进行的。要研究中学的教学效率, 最重 要的是找到一个合适的数学模型, 通过对选定评价指标 的综合分析和计算, 得出教学效率的结论。在国内外研 究的基础上, 选用数据包络分析 (DEA) 方法, 运用 “帕 累托最优” 思想对经济学中的生产效率进行评价, 从教 学投入和教学产出两个方面评价数学教学的有效性, 据 此找出在教学过程中存在的不足之处, 并对其加以改善 [4]

\section{2. 数据包络分析模型简介}

数据包络分析是数据包络分析是在 “相对效率评价” 概念的基础上发展起来的一种新的系统分析方法, 由 A. Charnes 和 W. W. Cooper 等人创建, 是一种效率评价 分析方法 ${ }^{[5]}$ DEA 方法将数学, 经济, 管理结合起来, 并 且在此基础上形成了一种新方法, 成为了研究同一类型 部门的相对有效性的一种有效工具, 成为我们设计分析 评价因素的重要模型体系和方法, 可以直接用于高校教 学质量评价和效率分析, 也可以用于多目标多方案的评 价和决策问题。

与传统评价方法相比, DEA 方法可以用来评价具有 多个输入，多个输出的的相同类型部门间的相对有效性, 故它极大的丰富了生产函数理论 ${ }^{[6]}$ 。同时 DEA 模型也在 经济分析和管理科学领域, 系统预测和决策分析领域, 评估技术，盈利能力，城市经济状况以及有效分配市场 等领域中起着重要作用。自从 1990 年代中期一些研究 人员将 DEA 方法引入教育评估领域以来, DEA 方法已在 我国的教育评估领域得到了广泛的应用。

在我们的研究体系中, 有 $\mathrm{n}$ 个研究对象, 称之为决 策单元, 每个决策单元都有 $\mathrm{m}$ 种类型的投入和 $\mathrm{s}$ 种类型 的产出, 我们希望投入越小越好, 输出越大越好。理论 上的“投入“表示该决策单元对”资源”的耗费; “产出”是 决策单元在消耗”资源”之后，表明”成效”的一些指标。

将决策单元 $j$ 记为 $D_{M U}(1 \leqslant j \leqslant n)$, 各决策单元 的输入和输出数据由图 1 给出:

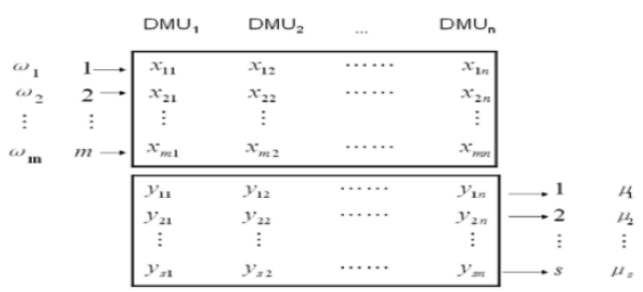

图 1: 输入输出数据图 $\boldsymbol{x}_{i j}$ 为第 $\mathrm{j}$ 个决策单元对第 $\mathrm{i}$ 种输入的投入量, $x_{i j}>0 ; y_{r j}$ 为第 $\mathrm{j}$ 个决策单元对第 $\mathrm{r}$ 种输出的产出率, $y_{r j}>0 ; w_{i}$ 为第 $\mathrm{j}$ 个决策单元的对第 $\mathrm{i}$ 种输入的一种度 量 (或称权); $u_{r}$ 为第 $\mathrm{j}$ 个决策单元对第 $r$ 种输出的一 种度量 (或称权) ${ }^{[7]}$; 为了方便, 在这里记

$$
\begin{aligned}
& x_{j}=\left(x_{1 j}, x_{2 j}, \cdots, x_{m j}\right)^{T}, j=1,2, \cdots, n, \\
& y_{j}=\left(y_{1 j}, y_{2 j}, \cdots, y_{s j}\right)^{T}, j=1,2, \cdots, n, \\
& w=\left(w_{1}, w_{2}, \cdots, w_{m}\right)^{T}, \boldsymbol{u}=\left(\boldsymbol{u}_{1}, \boldsymbol{u}_{2}, \cdots, \boldsymbol{u}_{s}\right)^{T}
\end{aligned}
$$

建立如下的分式规划模型

$$
\left\{\begin{array}{l}
\max \frac{u^{T} y_{0}}{v^{T} x_{0}} \\
\text { st. } \frac{u^{T} y_{j}}{v^{T} x_{j}} \leq 1, j=1,2, \cdots, n . \\
w \geq 0, \\
u \geq 0 .
\end{array}\right.
$$

其中, 权系数是 $r$ 维实数变量, $u$ 是 $s$ 维实数变量, 决策单元 $\mathrm{j}$ 的效率评价指数为

$$
h_{j}=\frac{\sum_{r=1}^{s} u_{r} y_{y j}}{\sum_{i=1}^{m} u_{i} x_{i j}}, j=1,2, \cdots, n .
$$

经过一系列的选择, 可使 $h_{j} \leq 1$ 对第 $j_{0}$ 个决策单元 的效率进行评价时, 此时以第 $\mathrm{j}_{0}$ 个决策单元的效率指数 作为指标, 约束条件为所有决策单元的效率指数, 构成 如下的模型:

$$
\left(\bar{P}_{C^{2} R}\right)\left\{\begin{array}{l}
\max \frac{u^{T} y_{0}}{v^{T} x_{0}}=V_{\bar{P}} \\
s t . \frac{u^{T} y_{j}}{v^{T} x_{j}} \leq 1, j=1,2, \cdots, n \\
w \geq 0 \\
u \geq 0
\end{array}\right.
$$

\section{3. 实例分析}

\section{1 数据的收集和处理}

在选择 DEA 模型进行分析计算时，应按照既定的原 则, 确定一套合理有效的投入和产出指标。选择投入和 产出指标时, 首先要满足我们的评价目的要求, 要能够 对评价对象做出客观的评价; 另外要求各评价指标之间 的线性无关性。最后需要考虑相对重要性以及指标的可 用性。若指标过多, 会导致评价难度增加, 可能出现每 个决策单元的有效性系数都会增加的情况, 可能接近 1 , 这就极大的降低了评价结果与决策单元的差异性, 因此 
决策单元的差异性程度下降。

\section{2 确定投入和产出指标} 学

基于相关理论, 初步选取某中学同一年级 10 名数

任课老师所在班级学生作为研究对象, 选取这 10 名教师作为评价对象。我们研究的评价指标包括反映教 学投入的指标, 反映教学产出的指标。我们将教师的学 历、教师的教龄、教师对多媒体软件的使用, 教师的进 修和培训时间、教师课堂教学评价、学生课余学习时间 等因素作为教学投入指标。产出指标因为无法直接主观 得出, 故通过诸如问卷调查等方法间接得到产出指标。 初始考虑产出指标为学生的理解能力、学生的创新能力、 学生对老师的教学满意度、班级毕业率、毕业平均分数 等。

由于实际操作及可测量性, 考虑到诸如理解能力等 指标难以测量, 教学满意度等指标更是存在着较大的不 确定性, 最终选取学生课余学习时间、教师的教龄、教 师的备课时间等 3 项指标作为教学投入指标。班级毕业 率, 毕业时班级数学平均成绩等 2 项指标作为教学产出 指标。

\section{3 决策单元的选择}

对某所初等学校而言, 不同的学科的学生培养成本 存在一定的客观差异, 输出指标也不一定相同, 因此不 同的学科也可以划分为不同类型的决策单元。由于同一 个学科存在多个班级, 班级之间学生人数, 学生资质, 学生成绩等存在差异, 所以不同班级同一学科也可以划 分为不同类型的决策单元。本文所研究的就是同一所学 校同一学科不同班级之间的效率评价, 在此处将数学这 一学科同一年级的不同班级的作为研究对象, 也相应的 选取其作为决策单元。

\section{4 数据处理}

确定了投入和产出指标之后, 基于相关理论, 收集 原始数据。直接采录某同学初三年级 10 个班级的数学 教师和所在班级学生的相关信息, 其中, 教师教龄 (a1), 教师备课时间 (a3), 班级毕业率 (b1), 毕业时班级数学 平均成绩 (b2) 等可直接得出, 学生课余学习时间 (a2) 则 通过问卷形式得出, 最终将数据进行汇总整理。其中班
级毕业率代表应届学生实际毕业人数与理论毕业人数 的百分比。

\section{5 结果分析}

表 1: 调查数据

\begin{tabular}{|l|l|l|l|l|l|}
\hline NOM & $\mathrm{b} 1$ & $\mathrm{~b} 2$ & $\mathrm{a} 1$ & $\mathrm{a} 2$ & $\mathrm{a} 3$ \\
\hline 1 & 1 & 88.2 & 7 & 123 & 60 \\
\hline 2 & 0.983 & 79.8 & 12 & 112 & 50 \\
\hline 3 & 1 & 86.3 & 20 & 100 & 50 \\
\hline 4 & 1 & 87.1 & 3 & 86 & 90 \\
\hline 5 & 1 & 89.2 & 25 & 76 & 45 \\
\hline 6 & 1 & 84.3 & 17 & 87 & 40 \\
\hline 7 & 0.992 & 90.1 & 15 & 102 & 60 \\
\hline 8 & 1 & 88.5 & 21 & 112 & 60 \\
\hline 9 & 1 & 86.9 & 16 & 132 & 70 \\
\hline 10 & 0.993 & 85.1 & 9 & 131 & 45 \\
\hline
\end{tabular}

根据求得的结果得出投入产出效率图如下图 2.

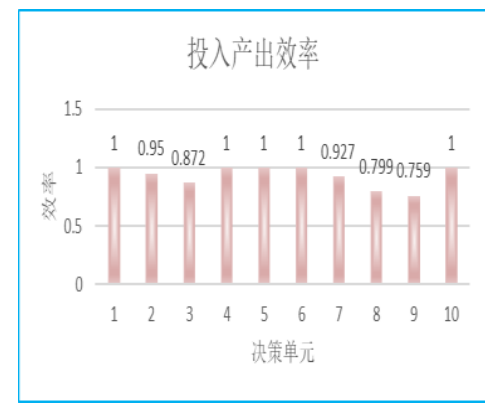

图 2: 投入产出效率图

结果表明, 总体教学效率为 $100 \%$ 的教师人数达到了 总参评人数的 $50 \%$, 效率为 $100 \%$ 则代表该评价单元的教 学投入和产出都达到最优状态, 达到了理想值, 或者说 达到了理论的帕累托最优。教学效率不足 $100 \%$ 的研究对 象, 理论上就没有达到帕雷托最优, 我们可以看到其中 效率最低的评价单元, 其效率仅为 $75.9 \%$, 表明该评价 单元的教学投入和产出总体上只达到理想值的 $75.9 \%$, 需要作出相应的改变才可以达到理想值。评价结果见下 表 2、表 3 .

表 2: 投入目标值

\begin{tabular}{|l|l|l|l|l|l|l|l|}
\hline DMU & 效率 & a1 & 占比 & a2 & 占比 & a3 & 占比 \\
\hline 1 & 1 & 7 & 1 & 123 & 1 & 60 & 1 \\
\hline 2 & 0.95 & 11.56 & 1 & 107.8 & 1 & 48.16 & 1 \\
\hline 3 & 0.87 & 17.81 & 1.03 & 89.06 & 1 & 44.53 & 1 \\
\hline
\end{tabular}




\begin{tabular}{|l|l|l|l|l|l|l|l|}
\hline 4 & 1 & 3 & 1 & 86 & 1 & 90 & 1 \\
\hline 5 & 1 & 25 & 1 & 76 & 1 & 45 & 1 \\
\hline 6 & 1 & 17 & 1 & 87 & 1 & 40 & 1 \\
\hline 7 & 0.93 & 15 & 1 & 102 & 1 & 60 & 1 \\
\hline 8 & 0.799 & 17.67 & 1.001 & 94.24 & 1.001 & 50.49 & 1.001 \\
\hline 9 & 0.759 & 12.31 & 1.005 & 101.6 & 1.005 & 53.89 & 1.005 \\
\hline 10 & 1 & 9 & 1 & 131 & 1 & 45 & 1 \\
\hline
\end{tabular}

表 3: 产出目标值

\begin{tabular}{|l|l|l|l|l|l|}
\hline DMU & 效率 & $\mathrm{b} 1$ & 占比 & $\mathrm{b} 2$ & 占比 \\
\hline 1 & 1 & 1 & 1 & 88.2 & 1 \\
\hline 2 & 0.95 & 0.99 & 1 & 85.0 & 0.999 \\
\hline 3 & 0.872 & 1 & 1 & 86.3 & 1 \\
\hline 4 & 1 & 1 & 1 & 87.1 & 1 \\
\hline 5 & 1 & 1 & 1 & 89.2 & 1 \\
\hline 6 & 1 & 1 & 1 & 84.3 & 1 \\
\hline 7 & 0.927 & 0.99 & 1 & 90.1 & 1 \\
\hline 8 & 0.799 & 1 & 1 & 88.5 & 1 \\
\hline 9 & 0.759 & 1 & 1 & 86.9 & 1 \\
\hline 10 & 1 & 0.99 & 1 & 85.1 & 1 \\
\hline
\end{tabular}

其中占比为目标值与实际值的比值。在所有达到最 优的决策单元里面, 其目标值与实际值之比为 1 , 也就 是总体教学效率为 $100 \%$, 代表着该研究对象的投入没有 咒余, 产出没有出现不足, 即达到最优。其余没有达到 帕累托最优的决策单元, 均表现为投入有所尣余, 投入 与产出没有达到最佳配比, 因此才出现了效率不足 1 的
情况。

针对我们得出的结果, 对没有达到最优的评价单元 按照比例进行相应增减, 调整之后再次进行计算。经过 两次调整之后, 发现所有的评价单元均达到了最优, 即 效率达到了百分之百。见下表 5 。

表 5: 效率图

\begin{tabular}{|l|l|l|l|}
\hline MMU & $\begin{array}{l}\text { 原始 } \\
\text { 效率 }\end{array}$ & $\begin{array}{l}\text { 修正后的 } \\
\text { 效率 }(1)\end{array}$ & $\begin{array}{l}\text { 修正后的 } \\
\text { 效率 }(2)\end{array}$ \\
\hline 1 & 1 & 1 & 1 \\
\hline 2 & 0.95 & 1 & 1 \\
\hline 3 & 0.872 & 1 & 1 \\
\hline 4 & 1 & 1 & 1 \\
\hline 5 & 1 & 1 & 1 \\
\hline 6 & 1 & 1 & 1 \\
\hline 7 & 0.927 & 1 & 1 \\
\hline 8 & 0.799 & 0.999 & 1 \\
\hline 9 & 0.759 & 0.995 & 1 \\
\hline 10 & 1 & 1 & 1 \\
\hline
\end{tabular}

由此可见, DEA 方法在我们的教学效率评价中有着 举足轻重的作用, 透过 DEA 方法, 我们可以看到, 一名 教师, 要想取得更好的教学效果, 不仅仅是经验的积累,
或者工作努力就能达到最理想的成绩的, 而是需要教师 和学生一起向着最合适的值努力。 


\section{4. 总结和建议}

本文把 DEA 模型用到教师教学水平的评价研究上, 把教与学的过程看作是一个投入产出系统，对 “教与 学” 水平进行有效性评价。教学评价的数据包络分析能 够获得传统评价方法得不到的许多有关教学能力、属性 与教学业绩之间关系的信息, 从而可以使学生和教师 更加有的放矢地采取措施提高教与学的水平, 促进教 育的良性发展。

\section{REFERENCES}

[1] Li N, Gao Y, Zhang Y.Research on the development teaching evaluation method based on DEA. JOURNAL of HUNAN AGRICULTURAL UNIVERSITY (SOCIAL SCIENCE EDITION - Quality Education Research), 2007,$11 ; 27-29$

[2] Shi Y. Practice and Thinking on the evaluation concept of developmental mathematics education. Mathematics learning and research, 2013,12:127

[3] Zheng X. New college entrance examination reform promotes the implementation of student development evaluation. Education and management, August 2019; 76-79

[4] Qin M, Li Z., Kong L..Reflection on the reform of PE teaching evaluation in Colleges and Universities Based on
DEA model. Educational exploration, sports science and technology literature bulletin, 2020, 28 (5): 75-76

[5] Wei Wang. Application of data envelopment analysis in the evaluation of input-output efficiency of middle school. Shanghai Education and research, 2016 (02): 52-56

[6] Wang C...Study on Evaluation of running efficiency of institutions of higher learning based on DEA. Hebei University of science and technology, 2010

[7] Deng X.,Li J., Zeng H.,Chen J.,Zhao J.. Analytic hierarchy process weight calculation method analysis and application research. Practice and understanding of mathematics, 2012,42 (07): 93-100

[8] Ma Z.. Data envelopment analysis model and method. Shanghai. Science Press. 2010.20-22

[9] Wang S..Research on education input and output . Shijiazhuang: Hebei Education Press, 1996:132

[10] Wei Q.. Data envelopment analysis (DEA). Science Bulletin, 2000 (17): 1793-1808

[11] Feldam K A. The Association between Student Ratings of Specified Instructional Dimensions and Extending the Syntheses of Data from Multisection Validity Students. Research in Higher Education, 1985, (1): 83. 